\title{
D.C. electrical conductivity measurements on ADP single crystals added with simple organic compounds
}

\author{
A ANNE ASSENCIA and C MAHADEVAN* \\ Physics Research Centre, S.T. Hindu College, Nagercoil 629 002, India
}

MS received 1 December 2003; revised 2 May 2005

\begin{abstract}
Pure and impurity added (with urea and thiourea) ADP single crystals were grown by the free evaporation method. D.C. electrical conductivity measurements were carried out along both the unique axis and perpendicular directions at various temperatures ranging from $40-150^{\circ} \mathrm{C}$ by the conventional two-probe method. Activation energies were also determined. The present study indicates that the conductivity increases with the increase in impurity concentration and temperature.
\end{abstract}

Keywords. Impurity added ADP crystals; density; electrical conductivity measurements.

\section{Introduction}

Ammonium dihydrogen orthophosphate (ADP), $\left(\mathrm{NH}_{4}\right)$ $\mathrm{H}_{2} \mathrm{PO}_{4}$, is an interesting material and, due to that fact, several research workers have shown considerable interest on it (Davey and Mullin 1976; Levina and Belyustin 1980; Nagalingam et al 1981; Ramesh and Mahadevan 1998).

The electrical conduction in dielectrics is mainly a defect controlled process in the low temperature region. The presence of impurities and vacancies mainly determine this region. The energy needed to form the defect is much larger than the energy needed for its drift. The conductivity of the crystal in the higher temperature region is determined by the intrinsic defects caused by thermal fluctuations in the crystal (Freeda and Mahadevan 2000).

The low temperature conduction region seems to be connected to mobility of vacancies. If the probability of occupation of an interstice is $f$, then the probability of finding a vacant neighbour site is $(1-f)$. Even for very high concentrations, of the order of $10^{20} \mathrm{~cm}^{-3}, f$ does not exceed $10^{-2}$ so that in real cases with concentration of interstitials of the order of $10^{15}-10^{20} \mathrm{~cm}^{-3},(1-f) \approx 1$ (Bunget and Popescu 1984).

The electrical conduction in $\left(\mathrm{NH}_{4}\right) \mathrm{H}_{2} \mathrm{PO}_{4}$ crystals can be understood as due to proton as in the case of $\mathrm{KH}_{2} \mathrm{PO}_{4}$. The conduction is mainly due to the anions, viz. $\left(\mathrm{H}_{2} \mathrm{PO}_{4}\right)^{-1}$ ions (Freeda and Mahadevan 2000; Priya et al 2001; Sancta et al 2001; Deepa et al 2002). Electrical conductivity may be determined by the proton transport within the framework of hydrogen bonds. The conductivity is associated with the incorporation into the crystal lattice of impurities and the formation of corresponding defects

*Author for correspondence (mahad@ sancharnet.in) in ionic crystals. The proton conduction may be accounted for by motion of protons accompanied by a $D$ defect (excess of positive charge). Migration of these defects may only modify electric polarization and may not change the charge at an electrode (Freeda and Mahadevan 2000). The motion of defects occurs by some kind of rotation in the bond with defects. The speed of displacement, $v \approx v a$, where $a$ and $v$ are the distance and frequency, respectively of the jump from one bond to the other.

It is a known fact that urea is a simple organic nonlinear optical material and thiourea is a simple organic ferroelectric material. Both urea and thiourea have no common ion with ADP. Hence, if added as an impurity into the lattice of ADP, replacement of either $\mathrm{NH}_{4}^{+}$or $\left(\mathrm{H}_{2} \mathrm{PO}_{4}\right)^{-}$ion is not possible. But, it is possible for these molecules to occupy some interstitial positions and create more defects and enhance the conductivity of ADP crystal. So, it is interesting to investigate the effect of these organic molecules as impurities on the electrical properties of ADP.

A research programme on the growth and physical properties of pure and impurity added ADP crystals is being carried out in this laboratory. As a part of the programme, we have studied the effect of urea and thiourea as impurities (added separately in the ADP solution used for the growth of crystals with impurity concentration ranging from $2000-10000 \mathrm{ppm}$, i.e. $0 \cdot 2-1 \cdot 0 \mathrm{~mole} \%$ ) on the d.c. electrical conductivity at various temperatures ranging from $40-150^{\circ} \mathrm{C}$ of ADP crystals grown by the free evaporation method. We report here the results obtained in our present study.

\section{Experimental}

Analytical reagent grade (AR) samples of $\left(\mathrm{NH}_{4}\right) \mathrm{H}_{2} \mathrm{PO}_{4}$, urea and thiourea along with double distilled water were 
used for the growth of single crystals by slow (free) evaporation method. ADP was added with urea and thiourea separately each in six different ADP: impurity molecular ratios, viz. $1: 0 \cdot 000$ (pure ADP), $1: 0 \cdot 002,1: 0 \cdot 004$, $1: 0 \cdot 006,1: 0 \cdot 008$ and $1: 0 \cdot 010$. The impurity was dissolved in $3.5 \mathrm{M}$ solution of ADP. Supersaturated aqueous solution of the salt $(3.5 \mathrm{M})$ was prepared in a $100 \mathrm{ml}$ beaker (corning glass vessel) and allowed to equilibrate at the desired temperature. The crystals were grown in unstirred condition. The temperature and volume were kept constant, respectively at $30^{\circ} \mathrm{C}$ and $20 \mathrm{ml}$ in all the crystal growth experiments. Small crystals appeared in the beginning due to slow evaporation and grew larger in considerable finite time. Best crystals (free from imperfections) were selected from this and used for measurements.

In order to understand qualitatively whether the added impurity has entered into the ADP lattice or not, we carried out the density measurements by using the flotation technique. Carbon tetrachloride of density, $1.594 \mathrm{~g} / \mathrm{cc}$ and bromoform of density, $2.890 \mathrm{~g} / \mathrm{cc}$, are respectively the lower density and higher density liquids, used.

As it was difficult to determine the impurity concentration in the crystal accurately without using sophisticated techniques, no experiment was carried out to determine this. However, we followed an approximate method available in the literature (Deepa et al 2002) to do the same.

Crystals with high transparency and large defect-free size $(>3 \mathrm{~mm})$ were selected and used for the d.c. electrical conductivity measurements. The extended portions of the crystals were removed completely and the opposite faces were polished and coated with good quality graphite to obtain a good ohmic contact.

The d.c. electrical conductivity measurements were carried out along both the unique axis $(c-)$ and perpendicular ( $a$ - and $b$-) directions for all the eleven crystals grown using the conventional two-probe technique using a million megohmmeter at various temperatures ranging from $40-150^{\circ} \mathrm{C}$ in a way similar to that followed by Freeda and Mahadevan (2000). The dimensions of the crystals were measured using a travelling microscope (L.C. =
$0.001 \mathrm{~cm})$. The conductivity $(\sigma)$ of the crystal was calculated using the relation

$$
\sigma=d /(R A)
$$

where $R$ is the measured resistance, $d$ the thickness of the sample crystal and $A$ the area of the face of the crystal in contact with the electrode. Inaccuracy involved in this measurement was within $\pm 4 \%$.

Plots between $\ln (\sigma)$ and $1000 / T$ were found to be very nearly linear. So, the conductivity values can be fitted to the relation

$$
\sigma=\sigma_{0} \exp [-E /(k T)]
$$

where $E$ is the activation energy, $k$ the Boltzmann constant, $T$ the absolute temperature and $\sigma_{0}$ the parameter depending on the material. Activation energies were estimated using the slopes of the above line plots [E=-(slope) $k \times 1000]$.

\section{Results and discussion}

Scalenohedral morphology was exhibited by all the crystals grown. All the crystals grown were found to be stable, colourless and transparent.

It was observed that the difference in densities of crystals grown in the same container was very small and negligible. Average densities are given in table 1 . The value observed for pure ADP $(1.805 \mathrm{~g} / \mathrm{cc})$ compares well with that reported in the literature $(1.803 \mathrm{~g} / \mathrm{cc})$ (John 1979). For both the impurities considered in the present study, the observed variation of density of ADP crystal caused by the impurities indicates that the impurities have entered into the lattice of ADP crystals. Moreover, it can be seen that the density varies further with the increase in impurity concentration of the aqueous solution of ADP used for the growth of crystals.

The estimated impurity concentration values are presented in table 1 . The observed result shows that the impurity in the crystal mainly occupies the interstitial positions.

Table 1. Average density values and estimated impurity concentrations.

\begin{tabular}{lccccc}
\hline & \multicolumn{2}{c}{ Density $(\mathrm{g} / \mathrm{cc})$} & & \multicolumn{2}{c}{$\begin{array}{c}\text { Estimated impurity concentration } \\
\text { in the crystal (mole\%) }\end{array}$} \\
\cline { 2 - 3 } \cline { 5 - 6 } $\begin{array}{l}\text { Impurity concentration in } \\
\text { the solution (mole\%) }\end{array}$ & $\begin{array}{c}\text { For urea } \\
\text { added ADP }\end{array}$ & $\begin{array}{c}\text { For thiourea } \\
\text { added ADP }\end{array}$ & & $\begin{array}{c}\text { For urea } \\
\text { added ADP }\end{array}$ & $\begin{array}{c}\text { For thiourea } \\
\text { added ADP }\end{array}$ \\
\hline Pure ADP & 1.805 & 1.805 & & - & - \\
0.2 & 1.788 & 1.827 & & 0.075 & 0.129 \\
0.4 & 1.784 & 1.820 & & 0.179 & 0.262 \\
0.6 & 1.777 & 1.811 & & 0.284 & 0.394 \\
0.8 & 1.775 & 1.809 & & 0.388 & 0.527 \\
1.0 & 1.772 & 1.789 & & 0.492 & 0.659 \\
\hline
\end{tabular}


The $\sigma$ values obtained along the two directions ( $a$ - and $b$-) perpendicular to the unique axis (c-direction) are, within experimental error, the same. The $\sigma$ values for the pure and impurity added ADP crystals are shown in figures $1-4$. The values of the activation energy, $E$, for the pure and impurity added ADP crystals are given in table 2 .

It can be seen that for both the impurities considered in the present study, the electrical conductivity increases with the increase in impurity concentration and temperature. This is similar to that observed for urea and thiourea added KDP (potassium dihydrogen orthophosphate) crystals (Priya et al 2001). The defect concentration will increase exponentially with temperature and consequently the electrical conduction also increases. The addition of impurity further increases the electrical conduction in the temperature region considered.

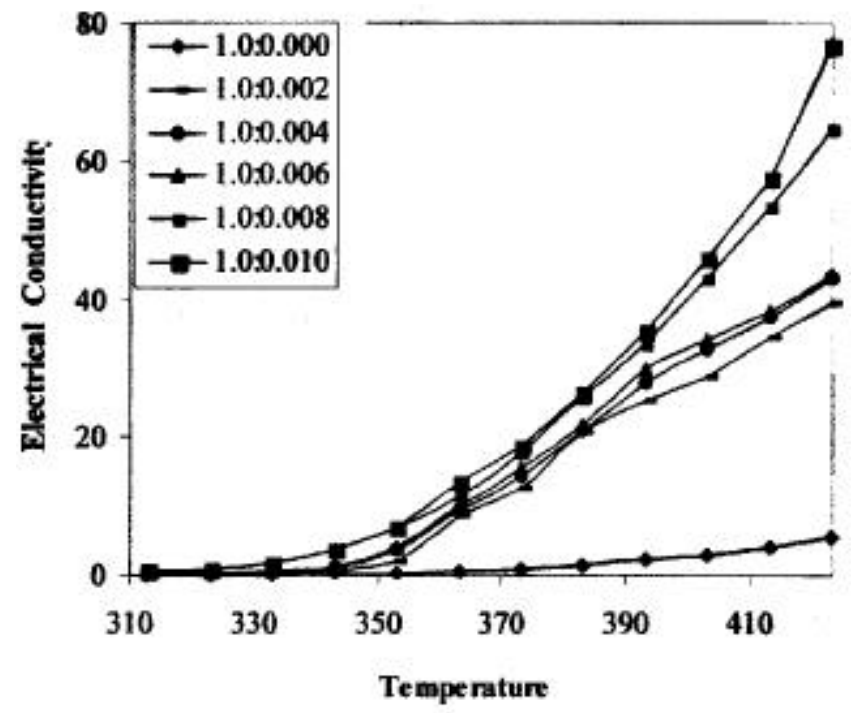

Figure 1. Electrical conductivities $\left(\times 10^{-6} \mathrm{mho} / \mathrm{m}\right)$ of pure and urea added ADP crystals along $a$-direction.

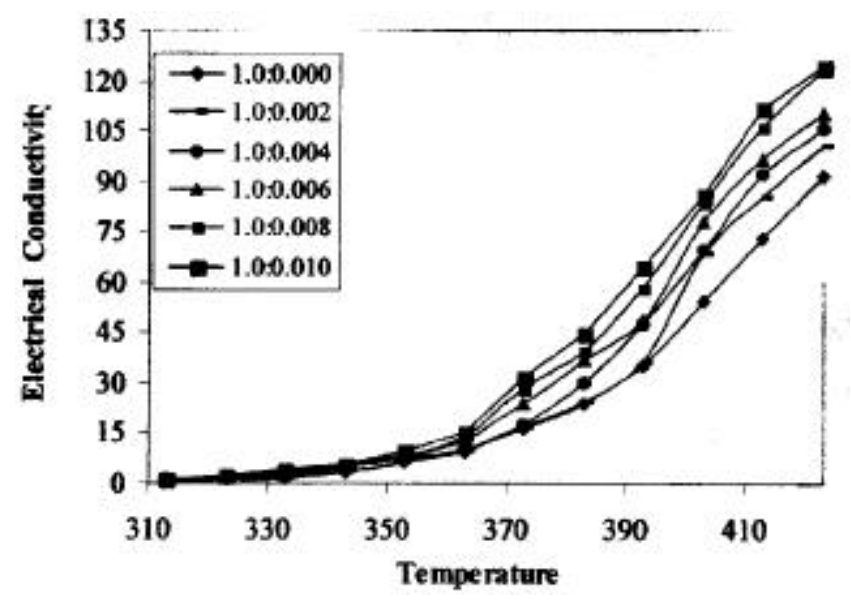

Figure 2. Electrical conductivities $\left(\times 10^{-6} \mathrm{mho} / \mathrm{m}\right)$ of pure and urea added ADP crystals along $c$-direction.
As the impurities mainly occupy the interstitial positions and the impurity concentrations considered in the present study are small, for impurity added ADP crystals, the impurity molecules can be assumed to be added in the ADP lattice in the same amount (ratio) as estimated. Hence, for impurity added ADP crystals, the total d.c. conductivity increases due to the addition of defects in the form of impurity molecules.

The conductivities are more along $c$-direction than those along $a$-direction for both the impurities considered in the present study. It may be stated that while the urea impurity is able to create more defects along $a$-direction, the thiourea impurity is able to create more defects along $c$-direction.

The present study gives further evidence to the statements that (i) the conduction in ADP is protonic (Dreyfus

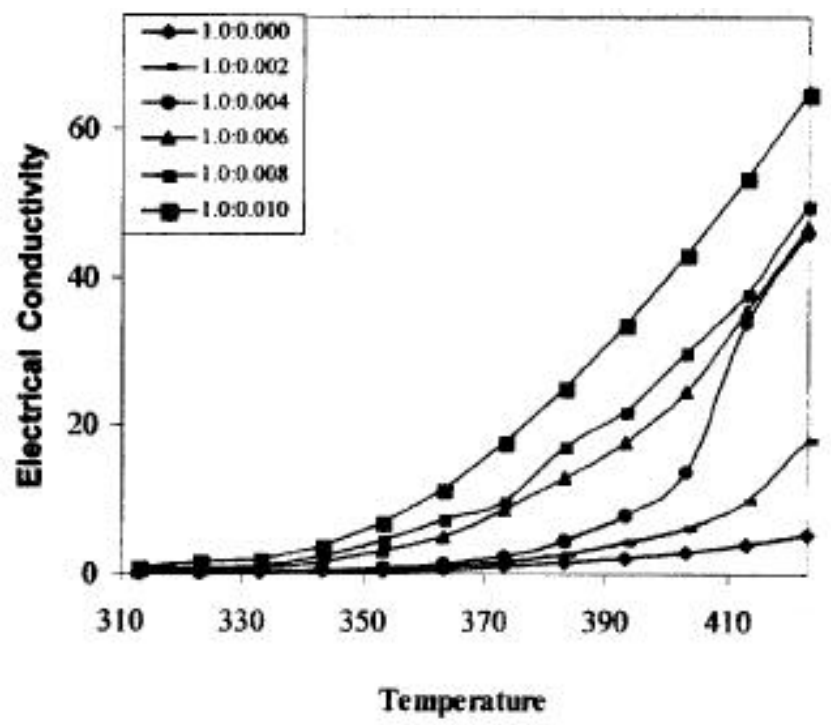

Figure 3. Electrical conductivities $\left(\times 10^{-6} \mathrm{mho} / \mathrm{m}\right)$ of pure and thiourea added ADP crystals along $a$-direction.

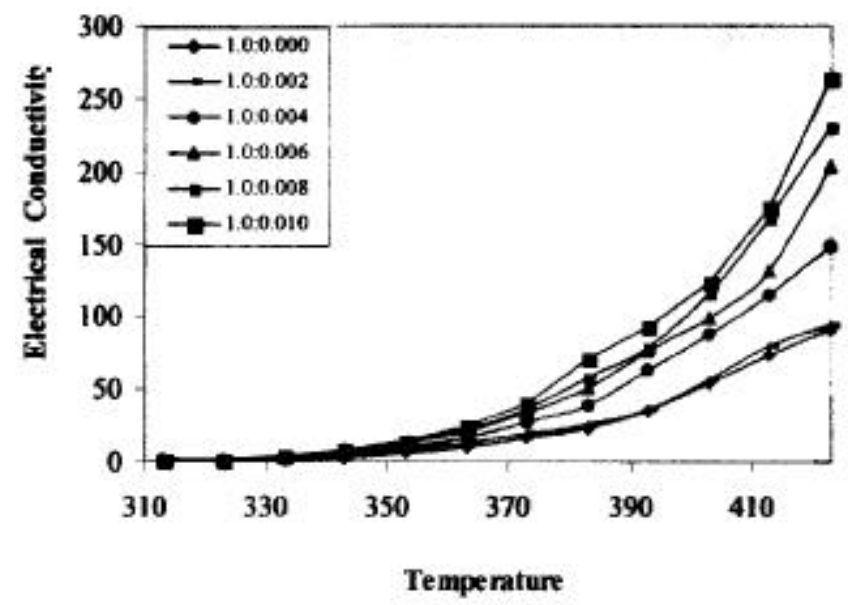

Figure 4. Electrical conductivities $\left(\times 10^{-6} \mathrm{mho} / \mathrm{m}\right)$ of pure and thiourea added ADP crystals along $c$-direction. 
Table 2. Activation energies.

\begin{tabular}{|c|c|c|c|c|}
\hline \multirow{3}{*}{$\begin{array}{l}\text { Impurity concentration in } \\
\text { the solution }(\text { mole } \%)\end{array}$} & \multicolumn{4}{|c|}{ Activation energy, $E(\mathrm{eV})$} \\
\hline & \multicolumn{2}{|c|}{ For urea added ADP } & \multicolumn{2}{|c|}{ For thiourea added ADP } \\
\hline & Along $a$-direction & Along $c$-direction & Along $a$-direction & Along $c$-direction \\
\hline Pure ADP & $0 \cdot 544$ & $0 \cdot 523$ & $0 \cdot 544$ & $0 \cdot 523$ \\
\hline $0 \cdot 2$ & 0.648 & 0.493 & $0 \cdot 613$ & 0.523 \\
\hline 0.4 & $0 \cdot 599$ & $0 \cdot 499$ & $0 \cdot 713$ & 0.544 \\
\hline 0.6 & $0 \cdot 592$ & $0 \cdot 505$ & 0.556 & 0.559 \\
\hline 0.8 & 0.513 & 0.509 & $0 \cdot 505$ & 0.566 \\
\hline $1 \cdot 0$ & $0 \cdot 525$ & $0 \cdot 507$ & $0 \cdot 472$ & $0 \cdot 581$ \\
\hline
\end{tabular}

and Nowick 1962; Silsbee et al 1964) and (ii) the conduction in ADP is mainly due to the anions, viz. $\left(\mathrm{H}_{2} \mathrm{PO}_{4}\right)^{-1}$ ions and not the cations, viz. $\left(\mathrm{NH}_{4}\right)^{+}$ions.

The activation energies are more along $a$-direction than those along $c$-direction for all the impurity concentrations in the case of urea added ADP crystals. However, there is no such variation observed in the case of thiourea added ADP crystals.

The $E$ values increase with the increase in impurity concentration in the case of thiourea along $c$-direction. However, there is no systematic variation observed in the case of thiourea added ADP along $a$-direction and in the case of urea added ADP along both $a$ - and $c$-directions.

The conduction region considered in the present study seems to be connected to mobility of vacancies. The low activation energies observed suggest that oxygen vacancies may be responsible for conduction in this region.

Also, the resistance measurement shows that the decomposition is not taking place at or less than $150^{\circ} \mathrm{C}$. So, the decomposition of ADP takes place at a higher temperature only.

\section{Conclusions}

Pure and impurity (urea and thiourea) added ADP single crystals were grown and d.c. electrical conductivities were measured along both the $a$ - and $c$-directions at various temperatures ranging from $40-150^{\circ} \mathrm{C}$. Density mea- surement indicates that the impurity molecules have entered into the lattice of ADP crystals. The present study gives further evidence to prove that the conduction in ADP is protonic and mainly due to the anions and not the cations. The present study indicates that the conductivity increases with the increase in impurity concentration and temperature in the temperature region considered.

\section{References}

Bunget I and Popescu M 1984 Physics of dielectrics (New York: Elsevier)

Davey R J and Mullin J W 1976 Krist. Technol. 1122

Deepa G, Freeda T H and Mahadevan C 2002 Indian J. Phys. A76 369

Dreyfus R W and Nowick A S 1962 Phys. Rev. 1264

Freeda T H and Mahadevan C 2000 Bull. Mater. Sci. 23335

John A Dean (Ed.) 1979 Lange's handbook of chemistry (New York: McGraw Hill Book Company)

Levina I M and Belyustin A V 1980 Soviet Phys. Cryst. 25209

Nagalingam S, Vasudevan S and Ramasamy P 1981 Cryst. Res. Technol. 16647

Priya M, Padma C M, Freeda T H, Mahadevan C and Balasingh C 2001 Bull. Mater. Sci. 24511

Ramesh R and Mahadevan C 1998 Bull. Mater. Sci. 21287

Sancta J A, Sutha A G, Freeda T H, Mahadevan C and Balasingh C 2001 Indian J. Phys. A75 245

Silsbee H D, Uebbing E A and Sehmidt V H 1964 Phys. Rev. A133 165 